\title{
Eficiência de trifloxysulfuron-sodium no controle de Cyperus rotundus L. na cultura da cana-de-açúcar
}

\author{
Anderson Ramos de Oliveira ${ }^{1}$ Silvério de Paiva Freitas² Ismael Lourenço de Jesus Freitas $^{3}$
}

\begin{abstract}
RESUMO
O objetivo foi avaliar a eficiência de trifloxysulfuron-sodium isolado, em mistura, ou em aplicação sequencial a outros herbicidas no controle de Cyperus rotundus L. na cultura da cana-de-açúcar. O estudo foi realizado em área de produção comercial do cultivar RB 72-454 na Usina Paraíso e em casa de vegetação da Universidade Estadual do Norte Fluminense Darcy Ribeiro, em Campos dos Goytacazes, Rio de Janeiro. As parcelas no campo foram submetidas a nove tratamentos: 2,4-D; trifloxysulfuron-sodium + ametryn; trifloxysulturon-sodium; 2,4-D + 2,4-D (20 dias após a primeira aplicação - DAP); 2,4-D + trifloxysulfuron-sodium + ametryn (20 DAP); 2,4-D + trifloxysulfuron-sodium (20 DAP); halosulfuron (20 DAP) e testemunha com e sem capina. Em casa de vegetação avaliou-se a viabilidade dos bulbos de C. rotundus em função dos tratamentos utilizados no experimento instalado em campo. Os resultados indicaram que o trifloxysulfuron-sodium isolado ou em mistura com ametryn, em aplicação sequencial ao 2,4-D, foi excelente no controle de C. rotundus. Esse herbicida e o halosulfuron, aplicados isoladamente, reduziram a capacidade de reinfestação da espécie daninha em razão da redução de sua propagação e do seu crescimento.
\end{abstract}

Palavras-chave: Controle químico, tiririca, herbicidas, Saccharum spp.

\section{ABSTRACT}

\section{Control of Cyperus rotundus L. with trifloxysulfuron-sodium in sugar-cane}

The aim of this study was to evaluate the efficiency of trifloxysulfuron-sodium used alone, mixed or in sequential application with other herbicides to control Cyperus rotundus L. in sugar cane. The study was conducted in an area of commercial production of variety RB 72-454 at "Paraíso Plant” and a greenhouse at the State University "Darcy Ribeiro”, Campos dos Goytacazes, RJ. The field plots were subjected to nine treatments: 2,4-D; trifloxysulfuronsodium + ametryn; trifloxysulturon-sodium, 2,4-D + 2,4-D (20 days after the first application - DAP) 2, 4-D + ametryn trifloxysulfuron-sodium (20 DAP), 2,4-D + trifloxysulfuron-sodium (20 DAP); halosulfuron (20 DAP), control with and without hand weeding. The greenhouse trial evaluated the viability of purple nutsedge bulbs as a function of the treatments used in the experiment carried out in the field. The results indicated that the trifloxysulfuron-sodium applied alone or mixed with ametryn in sequential application to 2,4-D was excellent in controlling C. rotundus. These herbicides, along with halosulfuron applied alone reduced the capacity for reinfestation of this specie by reducing propagation and growth.

Key words: Chemical control, herbicide, purple nutsedge, Saccharum spp.

\footnotetext{
Recebido para publicação em fevereiro de 2009 e aprovado em novembro de 2010

${ }^{1}$ Engenheiro-Agrônomo, Doutor. Embrapa Semiárido, BR 428, Km 152, CP 23, 56300-970, Petrolina, Pernambuco, Brasil. anderson.oliveira@cpatsa.embrapa.br

2 Engenheiro-Agrônomo, Doutor. Laboratório de Fitotecnia da Universidade Estadual do Norte Fluminense Darcy Ribeiro, Avenida Alberto Lamego, 2000, Horto, 28013-600, Campos dos Goytacazes, Rio de Janeiro, Brasil. silverio@uenf.br

${ }^{3}$ Engenheiro-Agrônomo, Mestre. Laboratório de Fitotecnia da Universidade Estadual do Norte Fluminense Darcy Ribeiro, Avenida Alberto Lamego, 2000, Horto, 28013-600, Campos dos Goytacazes, Rio de Janeiro, Brasil. ismaelljf@yahoo.com.br
} 


\section{INTRODUÇÃO}

O Brasil é o maior produtor mundial de cana-de-açúcar, seguido pela Î́ndia, China e Tailândia, e a produção alcançada pelo país representa quase dois terços da produção mundial. De acordo com dados do Ministério da Agricultura, Pecuária e Abastecimento (MAPA, 2009), a safra da cana-de-açúcar 2008/2009 foi de, aproximadamente, 564 milhões de toneladas. Em 2009, a área colhida foi de 8,6 milhões de hectares, com um rendimento médio de 80,2 t ha $^{-1}$ (IBGE, 2010). A produção de álcool na safra de 2008/2009 foi incrementada em, aproximadamente, cinco milhões de metros cúbicos em relação à safra anterior, alcançando 27,6 milhões de metros cúbicos deste combustível (MAPA, 2009). A estimativa é que ocorrerá um incremento superior a 1,5 bilhões de litros/ano de álcool hidratado e demanda estimada em 30 bilhões de litros até 2015 (MAPA, 2006) para atender à sociedade que busca combustíveis renováveis, menos poluentes e onerosos que os derivados do petróleo. Assim, é relevante aprimorar tecnologias de manejo de plantas daninhas na cultura a fim de garantir maior produtividade.

A cultura da cana-de-açúcar é muito afetada pela competição com as plantas daninhas, por apresentar, na maioria das situações, brotação e crescimento inicial lentos (Procópio et al., 2003), sendo o período crítico de competição variável e dependente de fatores relacionados à comunidade infestante (composição, densidade e distribuição), à própria cultura (espécie, cultivar, espaçamento entre os sulcos e densidade de plantio), à duração do período de convivência e à época em que esse ocorre. Em média, o período crítico de competição é de até 90 dias após a emergência da cultura (Kuva et al., 2001; Kuva et al., 2003), e para Cyperus rotundus a redução na produção da cultura ocorre a partir do $41^{\circ}$ dia após o plantio (Kuva et al., 2000).

Dentre as várias espécies de plantas daninhas presentes em canaviais brasileiros, $C$. rotundus é a de mais difícil controle, dado aos poucos herbicidas registrados para essa cultura com eficácia comprovada no seu controle, aliado às práticas culturais comuns à cana-de-açúcar, que provocam intensa mecanização do solo (Jakelaitis et al., 2003). No Brasil, essa planta daninha ocorre em praticamente todo o território, estimando-se a existência de um milhão de hectares infestados na cultura da canade-açúcar (Arévalo, 1996). Em razão de sua ramificada estrutura subterrânea e do eficiente sistema vegetativo de reprodução (bulbos e rizomas), C. rotundus pode provocar reduções de até $45 \%$ na produção de colmos quando presente em parte, ou no total, do ciclo produtivo da cultura (Keeley, 1987), em razão da competição por recursos do meio (água, luz e nutrientes) (Durigan, 2005). Além disso, apresenta alta eficiência fotossintética (Riemens et al., 2008) e mecanismos de dormência (Nesser et al., 1997).
Alguns estudos com Cyperus sp. têm identificado substâncias fitotóxicas altamente prejudiciais à cultura da cana, inibindo a germinação de gemas e provocando reduções no perfilhamento e na produção de colmos, causando falhas no canavial (Durigan, 1991).

A cana-de-açúcar é cultivada em áreas extensas, as plantas daninhas podem induzir reduções na produtividade e aumento de cerca de $30 \%$ dos custos de sua produção e de 15 a 25\% em cana-planta (Lorenzi, 1995). O controle químico de $C$. rotundus tem sido mais eficiente que o mecânico, sendo os herbicidas aplicados em préemergência, logo após o plantio, em área total e ou em pós-emergência, em aplicação dirigida ou em área total, conforme seletividade do produto para a cultura (Freitas et al., 2001). Dentre esses, os herbicidas do grupo químico das sulfonilureias (trifloxysulfuron-sodium e halosulfuron), que inibem a enzima acetolactato sintase (ALS), responsável pela síntese dos aminoácidos valina, leucina e isoleucina (McElroy et al., 2004), têm apresentado controle satisfatório (Brecke \& Unruh, 2000; Hudetz et al., 2000; Porterfield et al., 2002).

Sendo assim e considerando a importância do controle de C. rotundus em áreas canavieiras do Brasil, objetivouse avaliar a eficiência de trifloxysulfuron-sodium isolado, em mistura, ou em aplicação sequencial a outros herbicidas no controle desta planta daninha.

\section{MATERIAL E MÉTODOS}

Foram realizados dois experimentos, um em casa de vegetação e outro em condição de campo. Este último foi instalado em área de produção da cultivar RB 72-454, em ciclo de cana-soca de segundo corte com colheita realizada manualmente após a queima da planta, na Usina Paraíso, situada em Tocos, distrito do município de Campos dos Goytacazes, Rio de Janeiro, com altitude de $13 \mathrm{~m}$ acima do nível do mar, tendo como coordenadas geográficas $21^{\circ} 45^{\prime} 15^{\prime \prime}$ de latitude Sul e $41^{\circ} 19^{\prime} 28^{\prime \prime}$ longitude Oeste. O clima da região é classificado como Awi, com temperatura média de $24^{\circ} \mathrm{C}$ e precipitação anual de $905 \mathrm{~mm}$. O solo do local foi classificado como Cambissolo; argiloso, com pH (em $\mathrm{H}_{2} \mathrm{O}$ ) de 5,8; 27,5 g/kg de matéria orgânica; e teores de $\mathrm{Ca}^{2+}=40,8 \mathrm{mmol}_{\mathrm{c}} \mathrm{kg} ; \mathrm{Mg}^{2+}=56,8 \mathrm{mmol}_{\mathrm{c}} \mathrm{kg} ; \mathrm{K}^{+}=2,6 \mathrm{mmol}_{\mathrm{cl}}$ $\mathrm{kg} ;$ e P = 6,1 mmol $\mathrm{cg}$.

O delineamento experimental adotado foi em blocos casualizados, com nove tratamentos (Tabela 1) e quatro repetições, sendo as parcelas constituídas de cinco linhas da cultura, com cinco metros de comprimento, espaçadas 1,40 m entre si, correspondendo a uma área experimental total de $35 \mathrm{~m}^{2}$. A área útil de cada parcela foi equivalente a $16,8 \mathrm{~m}^{2}$, considerando como bordadura uma linha de plantio de cada lado da parcela e um metro de cada extremidade das três linhas centrais. 
Os herbicidas foram aplicados em pós-emergência das plantas daninhas e da cultura, com jato dirigido, utilizando-se pulverizador costal pressurizado a $\mathrm{CO}_{2}$, mantido à pressão constante de $3,5 \mathrm{kgf} \mathrm{cm}^{-2}$, provido de dois bicos de jato plano (tipo leque) 80.03, calibrado para aplicar o volume equivalente a $290 \mathrm{~L} \mathrm{ha}^{-1}$ de calda. Durante a aplicação dos herbicidas, realizada entre as 6h30min e 8h30min, com temperatura ambiente de $24^{\circ} \mathrm{C}$, umidade relativa do ar de $75 \%$, nebulosidade de $95 \%$ e velocidade do vento em torno de $6 \mathrm{~km} \mathrm{~h}^{-1}$.

A aplicação dos tratamentos constituídos somente de 2,4-D, trifloxysulfuron-sodium + ametryn ou trifloxysulfuron-sodium foi realizada quando as plantas daninhas estavam com aproximadamente $10 \mathrm{~cm}$ de altura (15 dias após a emergência). A aplicação sequencial foi feita 20 dias após a primeira pulverização (DAP). Na testemunha capinada o processo de remoção das plantas daninhas mediante capina manual foi realizado em três momentos: 30 dias antes da primeira aplicação dos herbicidas nas parcelas que seriam tratadas, no mesmo dia em que as parcelas destinadas a receberem os herbicidas foram tratadas e 30 dias após a primeira pulverização das parcelas que foram tratadas com herbicidas.

Foram realizadas avaliações de controle de C. rotundus e de produção de colmos de cana-de-açúcar. Para a determinação do controle da planta daninha foi utilizado um quadro vazado de $0,5 \mathrm{~m}$ de lado (área interna de $0,25 \mathrm{~m}^{2}$ ), onde as plantas de C. rotundus, delimitadas por essa área, foram contadas e coletadas, determinando-se a densidade de infestação, em função dos tratamentos utilizados, e o percentual de controle, este calculado em relação à testemunha sem capina. Os dados referentes à densidade de C. rotundus foram extrapolados para número de plantas por metro quadrado. A área experimental apresentava infestação de C. rotundus superior a 85\%, com densidade de, aproximadamente, 92 plantas por metro quadrado. Em cada parcela foram feitas quatro amostragens aos 30 e 90 dias após a aplicação dos tratamentos.

A colheita da cana-de-açúcar foi realizada quando a cana-soca atingiu 13 meses de idade. A produção de colmos das três linhas úteis da parcela foi determinada em uma balança portátil, sendo os resultados extrapolados e expressos em toneladas por hectare.

O segundo experimento foi instalado em casa de vegetação, em delineamento de blocos casualizados com quatro repetições, visando avaliar a eficiência dos herbicidas sobre a redução da viabilidade dos bulbos de $C$. rotundus. Aos 50 dias após a aplicação dos herbicidas (DAP), foram coletados os bulbos presentes à profundidade de 0 a $15 \mathrm{~cm}$ na área delimitada por um quadro metálico de $0,5 \mathrm{~m}$ de lado, o qual foi lançado aleatoriamente na área útil de cada parcela do experimento de campo. O solo foi peneirado e os bulbos separados, escolhendo-se, ao acaso, 40 (por tratamento), os quais foram plantados em vasos plásticos com capacidade de $8 \mathrm{~L}$ de substrato, na proporção de 10 bulbos por vaso. As plantas foram irrigadas duas vezes ao dia e mantidas por 41 dias na casa de vegetação. Ao final desse período, procedeu-se a coleta das partes aérea e subterrânea das plantas, sendo o material seco em estufa a $70{ }^{\circ} \mathrm{C}$, para determinação da biomassa seca da parte aérea e dos bulbos.

Os dados foram submetidos à análise de variância, aplicando-se o teste F. Para a comparação de médias de controle das plantas daninhas e de produtividade da canade-açúcar foi utilizado o teste de Tukey, a 5\% de probabilidade.

\section{RESULTADOS E DISCUSSÃO}

Aos 30 DAP verificou-se que as parcelas tratadas com trifloxysulfuron-sodium + ametryn, em aplicação sequencial ao 2,4-D, alcançaram nível de controle superior a 95\%, maior do que os tratamentos com aplicação isolada de trifloxysulfuron-sodium (56,25\%) e halosulfuron (57,50\%) (Tabela 2).

Aos 90 DAP, os tratamentos trifloxysulfuron-sodium + ametryn e trifloxysulfuron-sodium, em aplicação sequencial ao 2,4-D, e o halosulfuron isolado alcançaram mais de $85 \%$ de controle de C. rotundus. Estudos de-

Tabela 1. Tratamentos e doses utilizadas no experimento de controle de Cyperus rotundus em cultura da cana-de-açúcar RB 72-454

\begin{tabular}{lccc}
\hline & Tratamentos & & Doses utilizadas \\
\hline Aplicação inicial & 20 dias após a primeira aplicação & & Princípio ativo (g/ha) \\
\hline 2,4-D & - & $1.340,0$ \\
Trifloxysulfuron-sodium + ametryn & - & $37,0+1.463,0$ \\
Trifloxysulfuron-sodium & $2,4-\mathrm{D}$ & 15,0 \\
2,4-D & Trifloxysulfuron-sodium + ametryn & & $1.340,0+30+1.340,0$ \\
2,4-D & Trifloxysulfuron-sodium & $+37,0+1.463,0$ \\
2,4-D & Halosulfuron & $1.340,0+15,0$ \\
- & - & 113,0 \\
Testemunha com capina & - & - \\
Testemunha sem capina & & - \\
\hline
\end{tabular}

Rev. Ceres, Viçosa, v. 57, n.6, p. 736-741, nov/dez, 2010 
monstraram controle superior a $80 \%$ no controle de $C$. rotundus quando se utiliza o herbicida halosulfuron (Mascarenhas et al., 1995; Blum et al., 2000). Devido à alta capacidade de regeneração e multiplicação de $C$. rotundus (Nesser et al., 1997), o tratamento com trifloxysulfuron sodium isolado apresentou baixa eficiência de controle, igualando-se ao testemunha sem capina (Tabela 2).

Observa-se que as parcelas tratadas com halosulfuron e 2,4-D + trifloxysulfuron-sodium aumentaram a porcentagem de controle se comparada à da avaliação feita aos 30 dias após a aplicação (Tabela 2).

Ainda pela Tabela 2, verificou-se que as maiores produtividades de colmos de cana-de-açúcar foram obtidas nos tratamentos com trifloxysulfuron-sodium + ametryn, com 2,4-D + trifloxysulfuron-sodium + ametryn (20 DAP) e com o halosulfuron. O tratamento 2,4-D + trifloxysulfuron-sodium + ametryn, que proporcionou um dos melhores controles de C. rotundus, também se destacou em relação à produtividade, alcançando média superior a $90 \mathrm{t}$ $\mathrm{ha}^{-1}$. Os resultados indicaram que o trifloxysulfuronsodium isolado ou em mistura com ametryn, em aplicação sequencial ao 2,4-D, foi excelente no controle de $C$. rotundus, proporcionando produtividade de colmos superior em 18,4\% à testemunha capinada. Estes herbicidas, juntamente com o halosulfuron, aplicados isoladamente reduziram a capacidade de reinfestação da espécie daninha em razão da redução na propagação e no seu crescimento.

O 2,4-D, aplicado em concentrações altas, atua de maneira inversa à auxina natural (AIA) existente no interior das plantas, proporcionando distúrbios nos principais processos metabólicos (Ahrens, 1994). Esse herbicida paralisa o crescimento das manifestações epígeas de $C$. rotundus e inibe o desenvolvimento de rizomas laterais e bulbos, entretanto não evita novas brotações de bulbos. Por sua vez, o trifloxysulfuron-sodium apresenta amplo espectro de ação, podendo ser usado em reduzidas do- ses, com longo período residual no solo, o que garante o controle de plantas daninhas durante o crescimento inicial da cultura (Santos et al., 2004).

O halosulfuron destacou-se também em relação ao controle da tiririca aos 90 dias e em relação à produtividade das parcelas que foram submetidas a esse tratamento. Trabalhos científicos indicaram que a aplicação de 131,25 $\mathrm{g} \mathrm{ha}^{-1}$ de halosulfuron proporcionou controle de $C$. rotundus acima de $90 \%$, sendo seletivo à cana-de-açúcar, não havendo sintomas visuais de toxicidade ou redução do crescimento das plantas (Mascarenhas et al., 1995). Também foi observado em outros trabalhos que a aplicação de halosulfuron proporcionou controle de tiririca próximo a $90 \%$ aos 90 dias após o plantio da cana (Pessanha, 2000; Durigan et al., 2005).

No experimento em casa de vegetação, a biomassa seca da parte aérea das plantas de tiririca foi, principalmente, afetada pelos tratamentos com 2,4-D + 2,4-D em aplicação sequencial, 2,4-D + trifloxysulfuron-sodium + ametryn sequencial, 2,4-D + trifloxysulfuron-sodium sequencial e halosulfuron isolado. Observa-se que em relação ao sistema radicular uma das maiores biomassas encontradas foi no tratamento com testemunha capinada, evidenciando que apenas a retirada da parte aérea não caracteriza o controle efetivo da espécie invasora (Tabela 3).

Verifica-se, ainda, que a redução foi de aproximadamente $70 \%$ da massa seca dos bulbos, variando de 17,84 g no tratamento sem capina a 4,98 g no tratamento com 2,4-D + trifloxysulfuron-sodium + ametryn (Tabela 3). Tais resultados assemelham-se àqueles encontrados por outros pesquisadores (Durigan et al., 2004), que constataram reduções no número de bulbos quando se utilizou a mistura de trifloxysulfuron-sodium + ametryn nas doses de $1.312,5$ e $1.500 \mathrm{~g} \mathrm{ha}^{-1}$. A absorção radicular de trifloxysulfuron-sodium possibilita maior redução do crescimento de Cyperus rotundus e reduz a massa seca da parte aérea (McElroy et al., 2003).

Tabela 2. Controle de Cyperus rotundus aos 30 e 90 dias após aplicação dos tratamentos e produtividade da cana-de-açúcar

\begin{tabular}{|c|c|c|c|c|}
\hline \multicolumn{2}{|c|}{ Tratamentos } & \multicolumn{2}{|c|}{ Controle de C. rotundus (\%) } & \multirow{2}{*}{$\begin{array}{c}\text { Produtividade } \\
\text { (t/ha) }\end{array}$} \\
\hline Aplicação inicial & 20 dias após a primeira aplicação & 30 dias & 90 dias & \\
\hline $2,4-\mathrm{D}$ & - & $65,00 \mathrm{ab}$ & $60,00 \mathrm{abc}$ & 71,475 bc \\
\hline Trifl. sodium + ametryn & - & $70,00 \mathrm{ab}$ & $45,00 \mathrm{bc}$ & $87,142 \mathrm{ab}$ \\
\hline Trifloxysulfuron sodium & - & $56,25 \mathrm{~b}$ & $37,50 \mathrm{~cd}$ & $78,125 \mathrm{~b}$ \\
\hline $2,4-\mathrm{D}$ & $2,4-\mathrm{D}$ & $71,25 \mathrm{ab}$ & $55,00 \mathrm{abc}$ & $76,207 \mathrm{~b}$ \\
\hline $2,4-\mathrm{D}$ & Trifl. sodium + ametryn & 95,50 a & 91,25 a & 92,070 a \\
\hline 2,4-D & Trifl. sodium & $71,25 \mathrm{ab}$ & $90,00 \mathrm{a}$ & $80,050 \mathrm{~b}$ \\
\hline- & Halosulfuron & $57,50 \mathrm{~b}$ & $86,25 \mathrm{ab}$ & $85,385 a b$ \\
\hline Testemunha com capina & - & $72,50 \mathrm{ab}$ & 47,50 bc & $75,135 \mathrm{~b}$ \\
\hline Testemunha sem capina & - & $0,00 \mathrm{c}$ & $0,00 \mathrm{c}$ & 68,125 c \\
\hline
\end{tabular}

Médias seguidas pela mesma letra, na coluna, não diferem entre si pelo teste de Tukey a 5\% de probabilidade 
Tabela 3. Biomassa seca de plantas Cyperus rotundus em razão dos diferentes tratamentos

\begin{tabular}{lccc}
\hline & Tratamentos & Biomassa seca da & Biomassa seca \\
Aplicação inicial & $\begin{array}{c}\text { 20 dias após a } \\
\text { primeira aplicação }\end{array}$ & & $\begin{array}{c}\text { paérea (g) } \\
\text { dos bulbos (g) }\end{array}$ \\
\hline 2,4-D & - & $7,78 \mathrm{abc}$ & $10,43 \mathrm{ab}$ \\
Trifl. sodium + ametryn & - & $7,63 \mathrm{abcd}$ & $9,35 \mathrm{ab}$ \\
Trifloxysulfuron sodium & - & $7,74 \mathrm{abc}$ & $8,89 \mathrm{ab}$ \\
2,4-D & $2,4-\mathrm{D}$ & $5,31 \mathrm{bcde}$ & $6,13 \mathrm{~b}$ \\
2,4-D & Trifl. sodium + ametryn & $3,11 \mathrm{cde}$ & $4,98 \mathrm{~b}$ \\
2,4-D & Trifl. sodium & $2,55 \mathrm{de}$ & $3,68 \mathrm{~b}$ \\
- & Halosulfuron & $1,54 \mathrm{e}$ & $4,65 \mathrm{~b}$ \\
Testemunha com capina & - & $10,50 \mathrm{a}$ & $17,84 \mathrm{a}$ \\
Testemunha sem capina & - & $9,25 \mathrm{ab}$ & $13,25 \mathrm{ab}$ \\
\hline
\end{tabular}

Médias seguidas pela mesma letra, na coluna, não diferem entre si pelo teste de Tukey a $5 \%$ de probabilidade

\section{CONCLUSÕES}

O trifloxysulfuron-sodium isolado ou em mistura com ametryn, em aplicação sequencial ao 2,4-D, proporcionou excelente controle da C. rotundus. Estes tratamentos, juntamente com o herbicida halosulfuron aplicado isoladamente, reduziram o potencial de propagação de bulbos e o crescimento de C. rotundus; sendo, portanto, importante ferramenta para manejo integrado dessa espécie daninha na cultura.

\section{AGRADECIMENTOS}

À UENF (Universidade Estadual do Norte Fluminense), à FAPERJ (Fundação de Amparo à Pesquisa do estado do Rio de Janeiro) e à Syngenta, pelos incentivos e pelas condições para realização deste trabalho.

\section{REFERÊNCIAS}

Ahrens WH (1994) Herbicide handbook. 7.th. Champaign, Weed Science Society of America. 352p.

Arévalo RA (1996) Recentes avanços em controle químico de Cyperus rotundus (tiririca) em Saccharum spp. (cana-de-açúcar). In: Congresso Nacional da Sociedade dos Técnicos Açucareiros e Alcooleiros do Brasil - STAB, 6, Maceió. p.356-360.

Blum RR, Isgrigg III J \& Yelverton FH (2000) Purple (Cyperus rotundus) and yellow nutsedge (C. esculentus) control in bermudagrass (Cynodon dactylon) turf. Weed Technology, 14:357-365.

Brecke BJ \& Unruh JB (2000) CGA 362622 for torpedograss (Panicum repens) and purple nutsedge (Cyperus rotundus) control in bermudagrass. Proceeding South Weed Science Society, 53:228.

Durigan JC (1991) Manejo da tiririca (Cyperus rotundus L.) antes e durante a implantação da cultura da cana-de-açúcar (Saccharum spp.). Tese Livre-Docência. Universidade Estadual Paulista, Jaboticabal. 336p.

Durigan JC (2005) Effects of plant densities and management of purple nutsedge on sugarcane yield and effect of growth stages and main way of herbicides contact and absorption on the control of tubers. Journal of Environmental Science and Health, 40:111-117.
Durigan JC, Timossi PC \& Correia NM (2005) Densidades e manejo químico da tiririca na produtividade de cana-de-açúcar. Planta Daninha, 23:463-469.

Durigan JC, Timossi PC \& Leite GJ (2004) Controle químico da tiririca (Cyperus rotundus), com e sem cobertura do solo pela palha de cana-de-açúcar. Planta Daninha, 22:127-135.

Freitas SP, Coelho FC \& Pessanha HM (2001) Manejo de plantas daninhas na cultura da cana-de-açúcar na Região Norte Fluminense. Campos dos Goytacazes, UENF, 2001. 48p. (Boletim $\left.n^{\circ} 2\right)$.

Hudetz M, Foery W, Wells J \& Soares JE (2000) CGA 362622, a new low rate Novartis post-emergent herbicide for cotton and sugarcane. Proceeding South Weed Science Society, 53:163165.

IBGE - Instituto Brasileiro de Geografia e Estatística (2010) Confronto das safras de 2009 e das estimativas para 2010 - Brasil. Disponível em: http://www.ibge.gov.br/home/estatistica/indicadores /agropecuaria/lspa/defaulttab.shtm. Acessado em: 09 de junho de 2010.

Jakelaitis A, Ferreira LR, Silva AA, Agnes EL, Miranda GV \& Machado AFL (2003) Efeitos de sistemas de manejo sobre a população de tiririca. Planta Daninha, 21:89-95.

Keeley PE (1987) Interference and interation of purple and yellow nutsedges (C. rotundus and C. esculentus) with crops. Weed Technology, 1:74-81.

Kuva MA, Gravena R, Pitelli RA, Chistoffoleti PJ \& Alves PLCA (2001) Períodos de interferência das plantas daninhas na cultura da cana-de-açúcar. II - Capim-braquiária (Bracchiaria decumbens). Planta Daninha, 19:323-330.

Kuva MA, Gravena R, Pitelli RA, Chistoffoleti PJ \& Alves PLCA (2003) Períodos de interferência das plantas daninhas na cultura da cana-de-açúcar. III - Capim-braquiária (Bracchiaria decumbens) e capim-colonião (Panicum maximum). Planta Daninha, 21:37-44.

Kuva MA, Pitelli RA, Chistoffoleti PJ \& Alves PLCA (2000) Períodos de interferência das plantas daninhas na cultura da cana-de-açúcar. I - Tiririca. Planta Daninha, 18:241-251.

Lorenzi H (1995) Plantas daninhas na cultura da cana-de-açúcar: plantas daninhas na lavoura do nordeste brasileiro. In: $4^{\circ} \mathrm{En}-$ contro Técnico Goal, Cana-de-açúcar, Recife. Anais, Copersucar. p.50-58.

MAPA - Ministério da Agricultura, Pecuária e Abastecimento (2009) Anuário estatístico da agroenergia. Brasília, Ministério da Agricultura, Pecuária e Abastecimento/ACS. 160p. 
MAPA - Ministério da Agricultura, Pecuária e Abastecimento, Secretaria de Produção e Agroenergia (2006) Plano nacional de agroenergia. $2^{a}$ ed. rev. Brasília, Embrapa Informação Tecnológica, 110p.

Mascarenhas MTH, Galli AJB, Viana MCM, Macedo GAR \& Lara JFR (1995) Eficácia do halosulfuron no controle de tiririca (Cyperus rotundus L.) na cultura da cana-de-açúcar. Planta Daninha, 13:69-80.

McElroy JE, Yelverton FH, Burke IC \& Wilcut JW (2004) Absorption, translocation, and metabolism of halosulfuron and trifloxysulfuron in green kyllinga (Kyllinga brevifolia) and falsegreen kyllinga (K. gracillima). Weed Science, 52:704-710.

McElroy JE, Yelverton FH, Troxler SC \& Wilcut JW (2003) Selective exposure of yellow (Cyperus esculentus) and purple nutsedge (Cyperus rotundus) to postemergence treatments of CGA-362622, imazaquin, and MSMA. Weed Technology, $17: 554-559$

Nesser C, Aguero R \& Swanton CS (1997) Survival and dormancy of purple nutsedge (Cyperus rotundus) tubers. Weed Science, 45:784-790.
Pessanha HM (2000) Controle químico da tiririca (Cyperus rotundus L.) na cultura da cana-de-açúcar. Tese de Mestrado. Universidade Estadual do Norte Fluminense, Campos dos Goytacazes, 49p.

Porterfield D, Wilcut JW \& Askew DSD (2002) Weed management with CGA-362622, fluometuron, and prometryn in cotton. Weed Science, 50:642-647.

Procópio SO, Silva AA, Vargas L \& Ferreira FA (2003) Manejo de plantas daninhas na cultura da cana-de-açúcar. Viçosa, Universidade Federal de Viçosa, 150p.

Riemens MM, Weide RY \& Runia WT (2008) Nutsedge: biology and control of Cyperus rotundus and Cyperus esculentus - review of a literature survey. Wageningen, Plant Research International, 35p.

Santos JB, Procópio SO, Silva AA, Pires FR, Ribeiro Junior JI, Santos EA \& Ferreira LR (2004) Seletividade do herbicida trifloxysulfuron sodium para fins de fitorremediação. Revista Ceres, 51:129-141. 